\title{
X-ray measurement and analysis on parameters of intervertebral foramen in the lower lumbar spine associated with the superior ar- ticular process
}

\author{
Zhaoyang Qiu ${ }^{\mathrm{a}}$, Yang Yu, ${ }^{\mathrm{a},}$, ZhanYing Ma ${ }^{\mathrm{a}}$, Peng Yan ${ }^{\mathrm{a}}$, Yixuan Gao ${ }^{\mathrm{a}}$, Qixiang Wang ${ }^{\mathrm{a}}$ \\ ${ }^{a}$ Department of bone and spine surgery, Jinzhou medical university, Jinzhou, China.
}

\begin{abstract}
Objectives: In this study, we aimed to supplement the database of intervertebral foramen by measuring the parameters of the lower lumbar spine related to the articular process of the X-ray, which could lay a theoretical foundation for the individualized surgery of transforaminal endoscopic spine technique (TEST).

Methods: Anteroposterior and lateral X-rays of the lumbar spine from 104 adults ( 50 male and 54 female) who met the measurement criteria were selected. The height and width of the intervertebral foramen, the height of the intervertebral space, the length from the superior articular process (SAP) to the lower edge of the upper lumbar pedicle, and the length from SAP to posterior margin of superior vertebra posterior edge were measured. Results: There was no significant difference in the shape of the lower lumbar intervertebral foramen between normal adult men and women. There were significant differences in parameters related to the lower lumbar intervertebral foramen between the age group from 40 to 60 and above 60, and especially those related to the SAP.

Conclusion: Parameters of the lower lumbar intervertebral foramen were obtained from 104 normal adults, which supplemented the lumbar intervertebral foramen database. After the age of 60 , the morphology of the lower lumbar intervertebral foramen was significantly changed in normal adults, and especially parameters related to the SAP. This study provided a reference for individualized minimally invasive surgery using TEST for elderly people over 60 years of age.

Keywords: Transforaminal endoscopic spine technique; superior articular process; intervertebral foramen; lower lumbar spine; X-ray; age
\end{abstract}

\section{INTRODUCTION}

Symptomatic lumbar disc herniation (LDH) is one of the common causes of spinal surgery. Surgical treatment has evolved from traditional spine surgery to transforaminal percutaneous endoscopic discectomy (TPED) using the transforaminal endoscopic spine technique (TEST) ${ }^{[1]}$. TEST has several advantages such as less trauma, faster recovery, less scarring and more complete retention of the original physiological structure of the spine. And research showed that there was no statistical difference in clinical efficacy between spinal endoscopy and traditional open spinal surgery ${ }^{[2]}$.

\footnotetext{
*Corresponding author: Yang Yu

Mailing address: Department of bone and spine surgery, Jinzhou medical university, Jinzhou, China.

Email: doctoryoung@163.com

Received: 18 January 2020 Accepted: 09 April 2020
}

A large number of present studies have reported that TEST is a successful system operation, and the inserted cannula by transforaminal spinal endoscopy can safely pass through the intervertebral foramen ${ }^{[3-6]}$. With the emergence of the transforaminal technique, the importance of the study on the intervertebral foraminal morphology is self-evident, which has led to more research on the morphological characteristics of the intervertebral foramen ${ }^{[7-11]}$. Intervertebral foraminal morphology is particularly important for transforaminal spinal endoscopy. A natural hole composed of two adjacent vertebral pedicles contains nerves and blood vessels. The upper and lower boundaries are the upper vertebral vertebrae. Roof and floor are formed by inferior and superior notch of respective vertebral pedicles. The anterior boundary is composed of two vertebral discs and the posterior longitudinal ligament, and the posterior boundary is composed of the capsule of the small joint and part of the yellow ligament ${ }^{[12]}$. The shape of the intervertebral 
foramen is not constant, and some pathological changes will cause the morphologic change of the intervertebral foramen ${ }^{[13]}$. Many studies showed that the movement of the lumbar spine can also change of the morphology of the intervertebral foramen ${ }^{[14-16]}$. The study of intervertebral foraminal morphology has guiding significance for the imaging diagnosis and treatment of lumbar spine diseases. In terms of the technique of transforaminal spinal endoscopy, it is of guiding significance to grasp the parameters of intervertebral foramina for accurate insertion of cannula ${ }^{[17]}$.

The superior articular process (SAP) is an important part of the intervertebral foramen, and the morphology of the SAP is also an influencing important factor of intervertebral foramen stenosis and the nerve root compression. Intervertebral foramen stenosis was related to the degeneration of the intervertebral space and the upward movement of the SAP: the height of intervertebral space decreased, resulting in the height of intervertebral foramen reduced. The osteophyte formation of the articular process and the thickened of the ligament flava lead to the intervertebral foramen stenosis ${ }^{[7-11]}$. Hoogland et al. in 2006 proposed the transforaminal endoscopic spine system (TESSYS) technology, which is quite representative of TEST. The surgical procedure requires the use of a ring drill to grind the SAP in order to successfully place the cannula into the spinal canal indicating that the morphology of the SAP is an important factor for whether the cannula can enter the spinal canal in TESSYS technology. However, only a limited number of studies have included the intervertebral foramen, and even less has been done on the measurement of parameters associated with the SAP. In order to better grasp the geometry of the intervertebral foramen and improve the success rate of surgery, this study supplemented the database of the intervertebral foramen by measuring the parameters of the lower lumbar vertebra related to the SAP of the X-ray, which could provide a reference for the individualized surgery of TEST.

\section{METHODS AND MATERIALS}

\section{General information}

104 adults (50 male and 54 female) were selected from the outpatient and inpatient cases in the first affiliated hospital of Jinzhou Medical University who were to receive anteroposterior and lateral X-ray photographs of the lumbar spine from October 2017 to May 2019, all of whom were aged from 18 to 80 . Male age: $50.65 \pm 14.52$, female age: $49.63 \pm 18.04$.

Inclusion criteria:(1) No history of lumbar disease and lumbar trauma;(2) No history of waist and leg pain and lower extremity radiation pain;(3) No history of lumbar spine fractures and surgery;(4) No nerve rootsymptoms such as lower limb numbness and intermittent claudication;(5) The selected samples were taken in accordance with unified standards. No lumbar disc herniation, no lumbar spinal stenosis, lumbar pathological changes, osteogenesis, lumbar spondylolisthesis, scoliosis and other congenital or acquired osteopathy were confirmed as normal lumbar spine by X-ray imaging examination.

\section{Measurement methods and parameters}

The upright and lateral positions of the lumbar spine were photographed in the standing position. The projection centre was about $3 \mathrm{~cm}$ above the condyle, about $8 \mathrm{~cm}$ before the lumbar spinous process, about L3 vertebral level, and the projection distance was about 100 $\mathrm{cm}$, referring to the scale on the X-ray. The X-ray can clearly and completely show the shape of the intervertebral foramen and the SAP. The following parameters of each intervertebral foramen (13-14, 14-15, 15-s1) were measured and recorded on the X-ray of the lateral lumbar spine: (1) Determine the height of the intervertebral foramen in the lower lumbar vertebra (a). Use the measurement tool on the computer imaging software to make a line between the midpoint of the upper pedicle and the midpoint of the lower pedicle and read it. The value is the height of the intervertebral foramen (a); (2) Determine the width of the intervertebral foramen in the lower lumbar vertebra (b). For the superior lumbar pedicle, record the length from the posterior cortex of the lamina to the posterior cortex of the vertebral body, which is the width of the intervertebral foramen (b); (3) Determine the height of the intervertebral foramen in the lower lumbar spine (c). The length from the lower edge of the upper vertebrae to the upper edge of the lower vertebrae is the intervertebral height value (c); (4) Determine the distance from the SAP in the lower lumbar spine to the lower edge of the upper lumbar pedicle (d). The length from the apex of the SAP in the lower lumbar spine to the lower edge of the upper lumbar pedicle is d value;(5) Determine the distance from the SAP in the lower lumbar vertebrae to the posterior edge of the uppervertebra (e). The length from the apex of the superior articular process in the lower lumbar vertebrae to the posterior edge of the vertebral body is e value(Figure1).

\section{Statistical analysis}

The statistical software SPSS 21.0 was used for statistical analysis, and all results were represented as means \pm standard deviations. The obtained data were tested for normality, and the original data met the normality requirements of anova. The independent sample t-test was used to compare the differences caused by gender and age. When $\mathrm{P}<0.05$, the differences were considered statistically significant. 


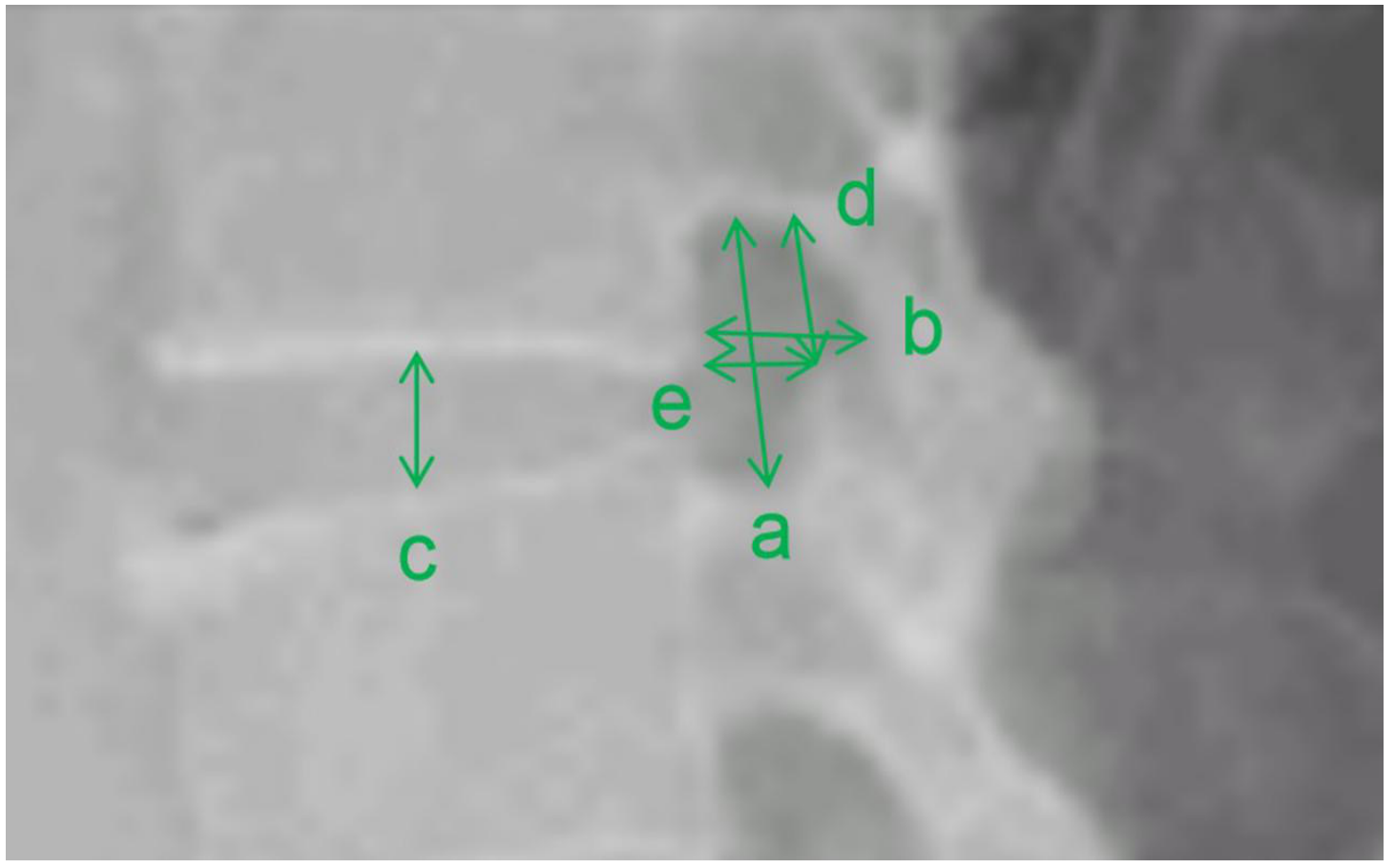

Figure 1. Diagram of intervertebral foramen measurement. (a) height of intervertebral foramen in a; (b) Width of intervertebral foramen; (c) Height of intervertebral space; (d) Distance between upper articular process and upper lumbar pedicle lower margin; (e) the distance between the upper articular process and the upper posterior margin of the vertebral body.

\section{RESULTS}

X-ray measurement of the lower lumbar intervertebral foramen in normal adults

A total of 300 lumbar X-rays (L3/4, L4/5, L5/S1) in 104 normal adults were measured and analyzed. The obtained data were all counting data, which were consistent with normal distribution by normal test. The relevant parameters of the lower lumbar foramen in normal adults were successively as follows: the heights of the intervertebral foramen were $22.25 \pm 2.55 \mathrm{~mm}$, $20.47 \pm 2.75 \mathrm{~mm}$, and $15.04 \pm 2.58 \mathrm{~mm}$; The widths of the intervertebral foramen were $9.78 \pm 2.06 \mathrm{~mm}$, $8.53 \pm 2.81 \mathrm{~mm}$, and $6.52 \pm 2.23 \mathrm{~mm}$; The heights of thelumbarintervertebral were $10.71 \pm 1.70 \mathrm{~mm}, 11.26 \pm 1.99 \mathrm{~mm}$, and $10.38 \pm 1.59 \mathrm{~mm}$; The lengths from SAP to the lower edge of the upper lumbar pedicle were $11.26 \pm 1.35 \mathrm{~mm}$, $10.34 \pm 1.44 \mathrm{~mm}$, and $7.61 \pm 1.32 \mathrm{~mm}$; The lengths from SAP to posterior margin of superior vertebra posterior edge were $6.84 \pm 1.45 \mathrm{~mm}, 5.96 \pm 1.96 \mathrm{~mm}, 4.56 \pm 1.56 \mathrm{~mm}$.

Parameters of the intervertebral foramen in the lower lumbar spine of different genders

The measured data of various parameters in the lower lumbar intervertebral foramen between different genders were shown in Table 1. The results showed that there was no significant difference in the parameters of the lower lumbar intervertebral foramen between different genders $(\mathrm{P}>0.05)$.

Table 1. X-ray measurement results of intervertebral foramen in normal adults of different genders $(\mathrm{x} \pm \mathrm{s}, \mathrm{mm})$.

\begin{tabular}{llll}
\hline Parameters & Male & Female & Total \\
\hline age & $50.65 \pm 14.52$ & $49.63 \pm 18.04$ & $50.14 \pm 16.30$ \\
L3-L4 a & $21.99 \pm 2.58$ & $22.51 \pm 2.52$ & $22.25 \pm 2.55$ \\
L3-L4 b & $10.06 \pm 2.00$ & $9.51 \pm 2.09$ & $9.78 \pm 2.06$ \\
L3-L4 c & $10.81 \pm 1.62$ & $10.61 \pm 1.78$ & $10.71 \pm 1.70$ \\
L3-L4 d & $11.16 \pm 1.41$ & $11.36 \pm 1.30$ & $11.26 \pm 1.35$ \\
L3-L4 e & $7.05 \pm 1.43$ & $6.63 \pm 1.46$ & $6.84 \pm 1.45$ \\
L4-L5 a & $20.34 \pm 2.56$ & $20.60 \pm 2.94$ & $20.47 \pm 2.75$ \\
L4-L5 b & $8.78 \pm 2.67$ & $8.28 \pm 2.96$ & $8.53 \pm 2.81$ \\
L4-L5 c & $11.41 \pm 2.00$ & $11.11 \pm 1.98$ & $11.26 \pm 1.99$ \\
L4-L5 d & $10.28 \pm 1.35$ & $10.41 \pm 1.53$ & $10.34 \pm 1.44$ \\
L4-L5 e & $6.14 \pm 1.85$ & $5.77 \pm 2.06$ & $5.96 \pm 1.96$ \\
L5-S1 a & $14.56 \pm 2.60$ & $15.52 \pm 2.50$ & $15.04 \pm 2.58$ \\
L5-S1 b & $6.47 \pm 2.21$ & $6.57 \pm 2.28$ & $6.52 \pm 2.23$ \\
L5-S1 c & $10.34 \pm 1.61$ & $10.42 \pm 1.58$ & $10.38 \pm 1.59$ \\
L5-S1 d & $7.38 \pm 1.35$ & $7.85 \pm 1.31$ & $7.61 \pm 1.32$ \\
L5-S1 e & $4.53 \pm 1.57$ & $4.59 \pm 1.57$ & $4.56 \pm 1.56$ \\
\hline
\end{tabular}


Parameters of the intervertebral foramen in the lower lumbar spine at different ages

The data of male and female groups were combined and counted, and a total of 104 cases were divided into three groups according to age: 28 cases under 40 years old, 41 cases between 40 and 60 years old, and 35 cases above 60 years old. A statistical comparison of the parameters in the lower lumbar intervertebral foramen among the three groups showed that there was a difference between the age group under 40 and the age group between 40 and 60 , but the difference was not statistically significant. There were significant differences in parameters a, c, $d$ and e between the age group from 40 to 60 years old and the age group above 60 years old (Table 2). Meanwhile, it was found that the differences in parameters related to the SAP were particularly significant, and the differences were statistically significant $(p<0.05)$.

\section{DISCUSSION}

Our results suggested that there were no significant differences between parameters in the lower lumbar intervertebral foramen between normal males and females. Gkasdaris G et al. found that no significant width differences for intervertebral foramen was observed at any level for either sex whether measured directly from the body or by CT ${ }^{[18,19]}$. This finding was also consistent with earlier reports on the sex-dependent size of the spinal dimension ${ }^{[20,21]}$.

There were differences in parameters a, c, d, and e between the age group from 40 to 60 years old and the age above 60 years old. Adams and Hutton ${ }^{[22]}$ found that the intervertebral joints were subjected to different stresses in different positions, and the lower the intervertebral space height, the more stress on the superior articular process, and the elder's lumbar superior articular process receives more stress than the youngers'. According to 'Wolf's Law' [23], bone is not an invariable material. It has the ability to reshape and repair after repeated damage under chronic external pressure for a long time in order to better adapt to the environment. As time goes on, these small changes gradually affect the anatomical structure of bones, ultimately leading to people over 60 show significant differences in intervertebral foramen parameters compared to those under 60 . With the improvement of living standards, the aging of China and the world's population will be inevitable. Therefore, our data could have clinical application on individualized minimally invasive surgery using transforaminal endoscopic spine technique for elderly people over 60 years of age. Our results also suggested that parameters related to the superior articular processes change more significantly. Many studies have reported that ${ }^{[7-11]}$ in-
Table 2. X-ray measurement results of intervertebral foramen in normal adults at different ages $(x \pm s, m m)$.

\begin{tabular}{|c|c|c|c|}
\hline Parameters & s Under 40 years old & $40-60$ years old & Above 60 years old \\
\hline L3-L4 a & $21.56 \pm 2.67$ & $22.51 \pm 2.40$ & $21.37 \pm 2.57$ \\
\hline L3-L4 b & $9.84 \pm 1.92$ & $9.98 \pm 2.05$ & $9.11 \pm 2.20$ \\
\hline L3-L4 c & $10.80 \pm 1.77$ & $11.12 \pm 1.52$ & $10.30 \pm 1.73$ \\
\hline L3-L4 d & $10.93 \pm 1.39$ & $11.38 \pm 1.27$ & $10.68 \pm 1.26$ \\
\hline L3-L4 e & $6.85 \pm 1.37$ & $7.02 \pm 1.43$ & $7.59 \pm 1.28$ \\
\hline L4-L5 a & $20.15 \pm 2.36$ & $20.77 \pm 2.78$ & $19.42 \pm 2.82$ \\
\hline L4-L5 b & $8.89 \pm 2.75$ & $8.40 \pm 2.99$ & $8.41 \pm 2.70$ \\
\hline L4-L5 c & $10.88 \pm 2.08$ & $11.37 \pm 2.02$ & $10.43 \pm 1.78$ \\
\hline L4-L5 d & $10.15 \pm 1.21$ & $10.52 \pm 1.48$ & $9.29 \pm 1.57$ \\
\hline L4-L5 e & $6.17 \pm 1.89$ & $5.87 \pm 2.09$ & $4.89 \pm 1.89$ \\
\hline L5-S1 a & $15.43 \pm 2.52$ & $15.13 \pm 2.62$ & $13.82 \pm 2.61$ \\
\hline L5-S1 b & $6.44 \pm 2.20$ & $6.49 \pm 2.47$ & $11.05 \pm 1.16$ \\
\hline L5-S1 c & $10.08 \pm 1.67$ & $10.72 \pm 1.57$ & $9.93 \pm 1.51$ \\
\hline L5-S1 d & $7.82 \pm 1.29$ & $7.63 \pm 1.34$ & $6.42 \pm 1.40$ \\
\hline L5-S1 e & $4.50 \pm 1.52$ & $4.54 \pm 1.75$ & $3.74 \pm 1.41$ \\
\hline
\end{tabular}

tervertebral foramen stenosis was related to the degeneration of the intervertebral space and upward movement of the superior articular process. The decrease of the intervertebral space height leads to a decrease in the height of the intervertebral foramen. The formation and hypertrophy of the facet joint capsule lead to the narrowing of the intervertebral foramen and compress the corresponding nerve roots. Due to factors such as degeneration of the intervertebral space and the movement of the articular processes, the height and width of the intervertebral foramen vary widely between reports. These may cause differences in the parameters of the lower lumbar intervertebral foramen between different ages. But we speculated that the changes in the two parameters related to the superior articular process in our results were associated to the decrease in the height of the intervertebral space. When the height of the intervertebral space decreased, it needs to further investigate whether the length from SAP to the lower edge of the upper lumbar pedicle (d) is more sensitive and representative than the intervertebral foraminal height. This study is only a part of the regional anatomy data obtained from the X-ray parameters of the lower lumbar intervertebral foramen associated with the SAP, and can merely provide a limited reference for the diagnosis and treatment of TEST and lumbar spinal stenosis. The specific application of our data to the guidance of intervertebral foraminal endoscopic surgery needs to be further studied and discussed.

\section{CONCLUSION}


Parameters of the lower lumbar intervertebral foramen were obtained from 104 normal adults, which supplemented the lumbar intervertebral foramen database. After the age of 60, the morphology of the lower lumbar intervertebral foramen was significantly changed in normal adults, and especially parameters related to the SAP. Our data could have clinical application on individualized minimally invasive surgery using TEST.

\section{DECLARATIONS}

\section{Financial support and sponsorship}

Funded by the Natural Science Foundation of Liaoning Province (No : 20180550920).

\section{Conflict of interest}

The author declares that there is no conflict of interest.

\section{REFERENCES}

1. Kapetanakis, S., Gkasdaris, G., Angoules, A. G., \& Givissis, P. (2017). Transforaminal percutaneous endoscopic discectomy using transforaminal endoscopic spine system technique: pitfalls that a beginner should avoid. World journal of orthopedics, 8(12), 874.

2. Telfeian, A. E., Iprenburg, M., \& Wagner, R. (2017). Endoscopic spine surgery: distance patients will travel for minimally invasive spine surgery. Pain physician, 20, E145-E149.

3. Choi, I., Ahn, J. O., So, W. S., Lee, S. J., Choi, I. J., \& Kim, H. (2013). Exiting root injury in transforaminal endoscopic discectomy: preoperative image considerations for safety. European Spine Journal, 22(11), 2481-2487.

4. Tsou, P. M., \& Yeung, A. T. (2002). Transforaminal endoscopic decompression for radiculopathy secondary to intracanal noncontained lumbar disc herniations: outcome and technique. The Spine Journal, 2(1), 41-48.

5. Ruetten, S., Komp, M., Merk, H., \& Godolias, G. (2008). Full-endoscopic interlaminar and transforaminal lumbar discectomy versus conventional microsurgical technique: a prospective, randomized, controlled study. Spine, 33(9), 931-939.

6. Yeung, A. T., \& Tsou, P. M. (2002). Posterolateral endoscopic excision for lumbar disc herniation: surgical technique, outcome, and complications in 307 consecutive cases. Spine, 27(7), 722-731.

7. Min, J. H., Kang, S. H., Lee, J. B., Cho, T. H., Suh, J. K., \& Rhyu, I. J. (2005). Morphometric analysis of the working zone for endoscopic lumbar discectomy. Clinical Spine Surgery, 18(2), 132-135.

8. Mirkovic, S. R., Schwartz, D. G., \& Glazier, K. D. (1995). Anatomic considerations in lumbar posterolateral percutaneous procedures. Spine, 20(18), 1965-1971.

9. Epstein, B. S., Epstein, J. A., \& Lavine, L. (1964). The effect of anatomic variations in the lumbar vertebrae and spinal canal on cauda equina and nerve root syndromes. The American journal of roentgenology, radium therapy, and nuclear medicine, 91, 1055.

10. Giles, L. G. (1994). A histological investigation of human lower lumbar intervertebral canal (foramen) dimensions. Journal of manipulative and physiological therapeutics, 17(1), 4-14.

11. Torun, F., Dolgun, H., Tuna, H., Attar, A., Uz, A., \& Erdem, A. (2006). Morphometric analysis of the roots and neural foramina of the lumbar vertebrae. Surgical neurology, 66(2), 148-151.

12. Choi, G., Pophale, C. S., Patel, B., \& Uniyal, P. (2017). Endoscopicspinesurgery.Journal of Korean Neurosurgical Society, 60(5), 485.

13. Bulyshchenko, G. G., Gaivoronskii, A. I., \& Gaivoronskii, I. V. (2018). Morphoscopic and Morphometric Characteristics of Intervertebral Foramina in the Lumbar Segment of the Spine. Neuroscience and Behavioral Physiology, 48(5), 582-587.

14. Revel, M., Mayoux-Benhamou, M. A., Aaron, C., \& Amor, B. (1988). Variations morphologiques des trous de conjugaison lombaires lors de la flexion-extension et de l'affaissement discal. Rev Rhum Mal Ostéoartic, 5, 361366.

15. Panjabi, M. M., Takata, K. O. I. C. H. I. R. O., \& Goel, V. K. (1983). Kinematics of lumbar intervertebral foramen. Spine, 8(4), 348-357.

16. Inufusa, A., An, H. S., Lim, T. H., Hasegawa, T., Haughton, V. M., \& Nowicki, B. H. (1996). Anatomic changes of the spinal canal and intervertebral foramen associated with flexion-extension movement. Spine, 21(21), 2412-2420.

17. Imran, J. B., Madni, T. D., Pruitt, J. H., Cornelius, C., Subramanian, M., Clark, A. T., ... \& Eastman, A. L. (2018). Can CT imaging of the chest, abdomen, and pelvis identify all vertebral injuries of the thoracolumbar spine without dedicated reformatting?. The American Journal of Surgery, 216(1), 52-55.

18. Gkasdaris, G., Tripsianis, G., Kotopoulos, K., \& Kapetanakis, S. (2016). Clinical anatomy and significance of the thoracic intervertebral foramen: A cadaveric study and review of the literature. Journal of craniovertebral junction \& spine, 7(4), 228.

19. GKASDARIS, G., HOURMOUZI, D., CHANIOTAKIS, C., HARITOUDIS, G., ASHRAFI, M. M., MOUSELIMIS, D., \& KAPETANAKIS, S. (2018). CT Assessment of the in vivo Osseous Lumbar Intervertebral Foramen: a Radiologic Study with Clinical Applications. Maedica, 13(4), 294.

20. Hasue, M., Kunogi, J. U. N. I. C. H. I., Konno, S. H. I. N. I. C. H. I., \& Kikuchi, S. (1989). Classification by position of dorsal root ganglia in the lumbosacral region. Spine, 14(11), 1261-1264.

21. Kikuchi, S. H. I. N. I. C. H. I., Hasue, M. I. T. S. U. O., Nishiyama, K. E. I. J. I., \& Ito, T. S. U. K. A. S. A. (1984). Anatomic and 
clinical studies of radicular symptoms. Spine, 9(1), 23-30.

22. Adams, M. A., \& Hutton, W. C. (1980). The effect of posture on the role of the apophysial joints in resisting intervertebral compressive forces. The Journal of bone and joint surgery. British volume, 62(3), 358-362.

23. Wolff, J. (1893). Das gesetz der transformation der knochen. DMW-Deutsche Medizinische Wochenschrift, 19(47), 1222-1224. 